\title{
Research on Forecast of Ultra-short-term Load under the Influence of Electric Railway
}

\author{
Ruifeng $\mathrm{An}^{1, \text { a }}$, Xinhui $\mathrm{Du}^{2, \mathrm{~b}}$, \\ Xiaodong Mi, Yuliang Ren, Jiming Wang \\ ${ }^{1,2}$ College of Electrical and Power engineering,Taiyuan University of Technology, Taiyuan, China \\ Shanxi Xinzhou Electric Power Supply Company, Shanxi Electric Power corporation, Xinzhou, \\ China \\ aemail: lovearf@163.com, bemail:394585211@qq.com
}

Keywords: ultrashort-term load forecast; wavelet theory; scaling function; support vector machine

\begin{abstract}
Railway is an important infrastructure of the state, national main artery and popular means of transport. With the gradually maturity of high-speed railway technology of China, the proportion of load of electric railway in the national power consumption load. As the electric railway load is characterized by high variation frequency, large fluctuation and poor periodicity, it causes a great influence on the forecast of local load. To solve this problem, this paper proposes a method of first treating the data by scale through wavelet analysis and then selecting partially similar day to forecast various loads in different frequencies with more load forecast models and improves wavelet svm forecast method to make the approximation under single scale in the past proceed in more scales and improve the partial approximation ability of this method. This method is used to forecast the ultrashort-term load of power system, the experiment result shows that the load forecast result precision of this method reaches above $97 \%$, effectively improving the forecast prevision of load under the influence of interference factors.
\end{abstract}

\section{Introduction}

As an important part of power system, power load forecast influences the planning, operation and management of power system. Improving the forecast precision has an important role to improve the safety and economics of the system. With the deep development of power marketization in the future, the power enterprises will improve the load forecast, especially ultrashort-term load forecast to a new height. While the ultrashort-term load forecast presents characteristics of large data to be measured and more complex situation by contrast to the short-term load forecast and have higher requirements to the forecast precision and speed.

Under this background, in this paper, we forecast the load with the grid supply load of a city in Shanxi to explore the ultra short-term load forecast method under the influence of electric railway. As local basic load value is relatively small, electric railway is fluctuated severely without obvious law, making the proportion of electric railway load in the total load increase, which directly influences the forecast precision. Against this situation, according to the characteristics of ultrashort-term load forecast, we introduce the wavelet analysis method in signature in the load forecast in this paper, decompose the data to be measured according to different time-frequency information and amplitudes, get different levels of approximate signals and detailed signals after decomposition and then conduct load forecast to the two kinds of signals respectively.

When analyzing the similar day and similar curve of the data, we introduce the wavelet support vector machine forecast mentioned in forecast [5]. Due to limited approximation capability of single-scale wavelet core in the model training, in order to overcome this defect, a more-scale approximation function is introduced to improve the approximation effect. 


\section{Analysis on electric railway load}

With the development economy and technology, railway has become a large consumer in the national energy consumption. For the advantages of performance and primary energy consumption, electric railway has gradually replaced the railway line dragged with steam locomotive or diesel locomotive. With the maturity of high-speed railway technology, the power consumption of electric railway has been further improved, so its influence on the power grid is also more and more obvious.

The city researched in this paper, for its important traffic and geographical position, is characterized by rapid development speed of electric railway, but other industries are relatively backward. The unbalanced industrial development causes that in the power load, the proportion of electric railway load in the grid supply load is large, about $15 \%-17 \%$. While electric railway is an important guarantee for the development of national economy, its operation method is flexible, but limited by the current detection method, making it is unable to detect the accurate operation law, as shown in Figure 1.
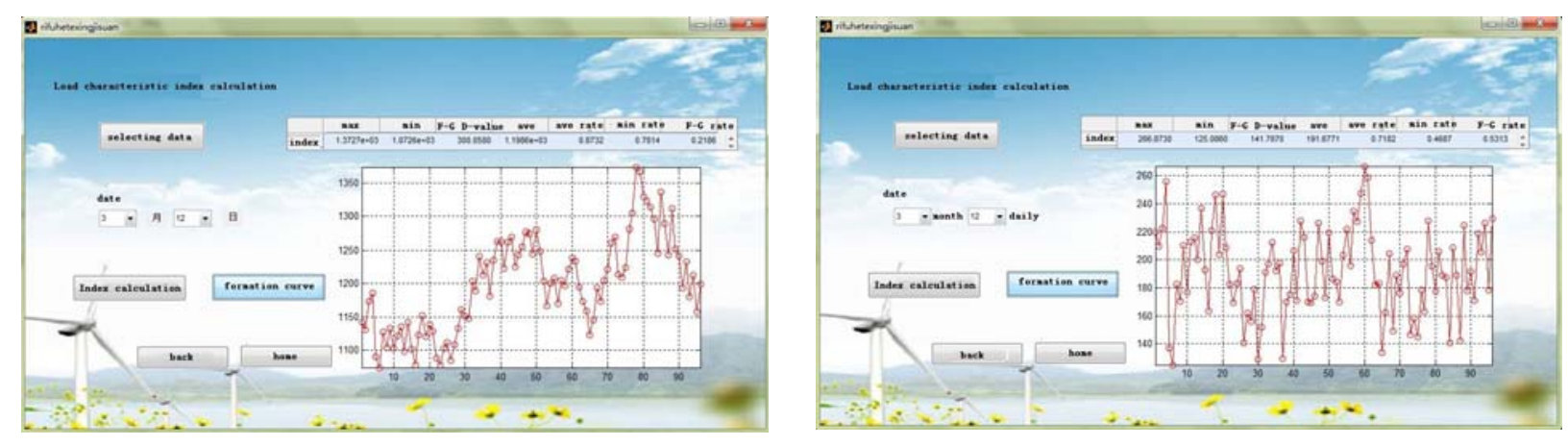

Fig.1. Screenshot of daily load indicator calculation software

Figure 1 is the screenshot of using matlab self-programming software-daily load characteristic indicator calculation module to calculate the local power grid supply load and electric railway load operation on March 12, 2012. As shown in Figure 1(a), the daily curve of power-grid load has obvious peak and valley characteristics, but in Figure 1(b), there are more data exceeding 50\% continuously occurring at the daily load curve of electric railway, which can be seen as an unpredictable load with out obvious cycle theoretically. In the load value, the average load value on the day of grid supply load is $1198.6 \mathrm{MW}$, and the average value on the day of electric railway load is $191.7 \mathrm{MW}$, accounting for $15.9 \%$ of the total load. It can be seen that although electric railway cannot change the trend of grid supply load thoroughly, it directly influences the smooth degree of load curve, and even become the main factor of load change partially, having quite an obvious influence on the load forecast result. Relatively, the traditional forecast method has a large error by contrast to such load. In order to reduce such influence of electric railway on the load forecast, we will introduce multi-scale wavelet analysis theory in this paper to process the load signal.

\section{Power load wavelet analysis}

As electric railway is characterized by complex composition and large influence of artificial factors on its operation condition, it is unrealistic to analyze its operation law objectively. Therefore, it is expected to control the main factors of variation through wavelet analysis theory in this paper to reduce the influence of irregular factors on the load variation.

Wavelet transformation is the result of development and evolution of windowing Fourier transformation (WFT), which can takes into account of the analysis of time field and frequency field on the signal in signal processing. In this paper, through wavelet analysis ,we use scaling 
function to distinguish the loads in different frequencies and amplitudes and decompose the input signal into detailed part and approximation part, and then further decompose the large-scale approximation part, by such repetition, the approximation part and detailed part in any scale can be obtained, as shown in Figure 2 and Figure 3.

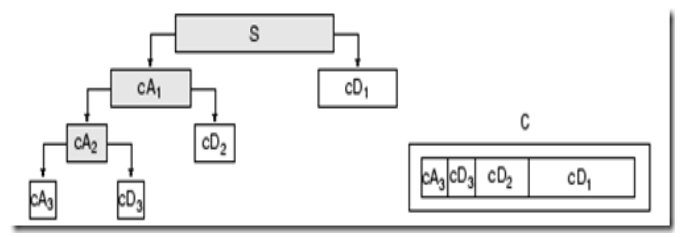

Fig.2. Three-layer signal decomposition
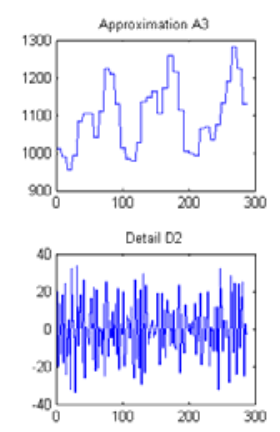

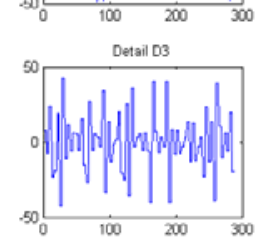

Fig.3. Wavelet signal reconstruction

Figure 2 shows the three-layer decomposition to the input signal, in which cA3 is the third layer of approximation part of the signal, $\mathrm{cD} 1, \mathrm{cD} 2$ and $\mathrm{cD} 3$ are respectively the first, second and third layers of detailed parts of the signal.

Figure 3 shows the three-layer signal decomposition and reconstruction by using the wavelet tool kit in matlab to select 3 days' power load continuously at random. A3 is the approximation signal and D3, D2 and D1 are respectively the detailed signals in different scales.

The analysis result shows that approximation signal A3 has a relatively strong regularity, this is because the approximation signal reflects the low-frequency part of the signal, which reflects the variation of main compositions of the signal and has a strong regularity. By contrast, the detailed signal of each scale has certain periodicity, but the periodic laws is often less than 24h. Meanwhile, the signal also contains many aperiodic components, which have higher requirements to the forecast model.

Next, we will verify the load forecast on the basis of wavelet decomposition and reconstruction and in combination with the research result of existing ultrashort-term load forecast (similar day judgment and forecast model setup).

\section{The selection of local similar day}

Selecting similar day and similar curve in the ultrashort-term load forecast has a direct influence on the training of load model, so it has an important significance to improve the load forecast. In this paper, we respectively analyze and process the similar day and similar curve on the basis of wavelet analysis.

When selecting the similar day, it is required to select the range of similar historical day. In consideration of the time interval and season in selection of similar day, the historical data two months before the forecast day and one month before and after the same day of the previous year of the forecast day as the sample data. The similar day data include value similarity and form similarity, as shown in Figure 4.

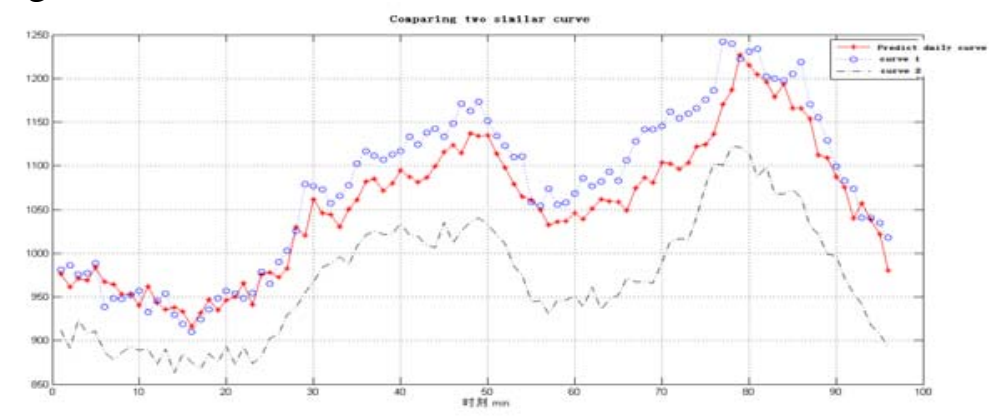

Fig.4. Two different types of similar days

The forecast day curve and curves 1 and 2 in the figure are selected from the power load curve 
diagram of a place on March 20, 21, and February 21, 2012. As shown in the figure, curve 1 and the objective curve have a very strong similarity, although curve 2 has a large value gap, it has the similar trend, both curves should be considered during forecast. Maintaining the Integrity of the Specifications

Besides, it is also required to analyze the partial sequence characteristics of the load and introduce them into the forecast algorithm, which is beneficial for improve the forecast precision. Therefore, the shape coefficient mentioned in reference [7] is introduced to calculate the partial similarity of the curve.

$$
S_{i T}=\frac{1}{N} \sum\left(x_{i T}-e_{i T}\right)
$$

Where, ${ }^{X}{ }_{i T}$ is the partial sequence of similar day, ${ }_{i T}$ is the partial sequence of forecast day and

$\mathrm{N}$ is the number of sequence element.

If one sequence and another one are more approximate to the translation state in vertical direction, then the shape coefficient ${ }^{S_{i T}}$ is more approximate to 0 ; the more different the two sequences in shape is, the larger ${ }^{{ }{ } T}$ will be. Therefore, ${ }^{S_{T T}}$ can reflect the shape nature difference of the two sequences very well.

By comparing the shape coefficient ${ }^{S_{i T}}$, we can the partial most similar day. The threshold $\mathrm{w}$ is set based on the minimum shape coefficient ${ }^{S_{i T}}$ and the load curve meeting the conditions in the similar range as the similar day.

\section{Multi-scale wavelet kernel support vector machine}

The setup of model is an important part of the load forecast and is the key point concerning the success of forecast. After processing the original load in various ways, we select the relatively mature and widely used SVM intelligent forecast method.

Multi-scale wavelet kernel support vector regression method has a relatively strong robustness and good generalization ability, while the selection of this model is the key point to obtain the good generalization ability, in which the complexity of adopting multi-scale parameter selection is larger

$$
k\left(x, x^{T}\right)=k\left(x-x^{T}\right)=\prod_{i=1}^{d} h\left(\frac{x_{i}-x_{i}^{T}}{a_{i}}\right)=\prod_{i=1}^{d} \cos \left(1.75 \times \frac{x_{i}-x_{i}^{T}}{a_{i}^{T}}\right) \exp \left(-\frac{\left(x_{i}-x_{i}^{T}\right)^{2}}{2 a_{i}^{2}}\right) \begin{array}{r}
\text { than } \begin{array}{r}
\text { the } \\
\text { single } \\
\text { kernel }
\end{array} \\
\text { parameter }
\end{array}
$$

selection. In order to improve the approximation capability of the multi-scale function, the support vector in this paper is of Smrlet wavelet kernel, $\mathrm{h}(\mathrm{x})$ in formula (2) is its mother wave and ${ }^{k}\left(x, x^{T}\right)$ is its corresponding kernel function.

$$
h(x)=\cos (1.75 \times x) \exp \left(-\frac{x^{2}}{2}\right)
$$

Where, $x_{i}, x_{i}^{T}, a_{i} \in R ; a_{i} \neq 0 ; x, x_{i}^{T} \in R^{d}$

Give the sample set $\left\{\left(x_{i}, y_{i}\right)\right\}_{i=1}^{n}, x_{i} \in R^{d}, y_{i} \in R$. Respectively regress the sample set in two different sample scale, the scales are respectively set as scale 1 and scale 2 and it is assumed that the scale factor of wavelet kernel function $\mathrm{k} 1$ of scale 1 is larger than the scale factor of kernel function $\mathrm{k} 2$ corresponding to scale 2. LS-WSVM on scale 1 is the approximation to the objective function on the coarse function, while that on scale 2 is the approximation to the sample residual of LS-WSVM on scale 1.

According to the principle of least squares support vector machine, assume that the regression 
model of wavelet support vector machine on scale 1 to the sample set is:

$$
f(x)=f_{1}(x)+f_{2}(x)=w_{1} \phi_{1}(x)+w_{2} \phi_{2}(x)+b \quad f_{1}(x)=w_{1} \phi_{1}(x)+b_{1}
$$

While the regression model to the sample residual error $\left\{\left(x_{i}, y_{i}-f_{1}\left(x_{i}\right)\right)\right\}_{i=1}^{n}$ on scale 2 is

$$
f_{2}(x)=W_{2} \phi_{2}(x)+b_{2}
$$

Where, $\Omega_{1 i 1}=\phi_{1}(x), \phi_{1}(\bullet)$ and $\phi_{2}(\bullet)$ are respectively corresponding to the characteristic mappings of wavelet kernel function on scale 1 and scale 2, i.e.

$$
k_{1}\left(x, x_{i}\right)=\left(\phi_{1}(x), \phi_{1}(x)\right) \ldots k_{2}\left(x, x_{i}\right)=\left(\phi_{2}(x), \phi_{2}(x)\right)
$$

Then, the final multi-scale least squares wavelet support vector machine model is:

$$
\text { Where, } b=b_{1}+b_{2} \text {. }
$$

\section{Example simulation and analysis}

Against the problem that electric railway influences the power grid supply load forecast, in combination with the wavelet analysis theory, historical similarity distinguishing and an improved SVM forecast method, we forecast and analyzed the load in a city in this paper. The data are taken from the daily load data of a city in Shanxi in 2011 and 2012 provided by the cooperative unit, which are real and reliable.

In this paper, we first calculate the similar day and similar curve of two signals after wavelet decomposition reconstruction, after determining the training sample, we use multi-scale SVM model for forecast and finally superimpose of forecast results of the two signal linearly to obtain the final forecast result, by comparing the two tradition methods, we obtain the forecast advantages of this method.
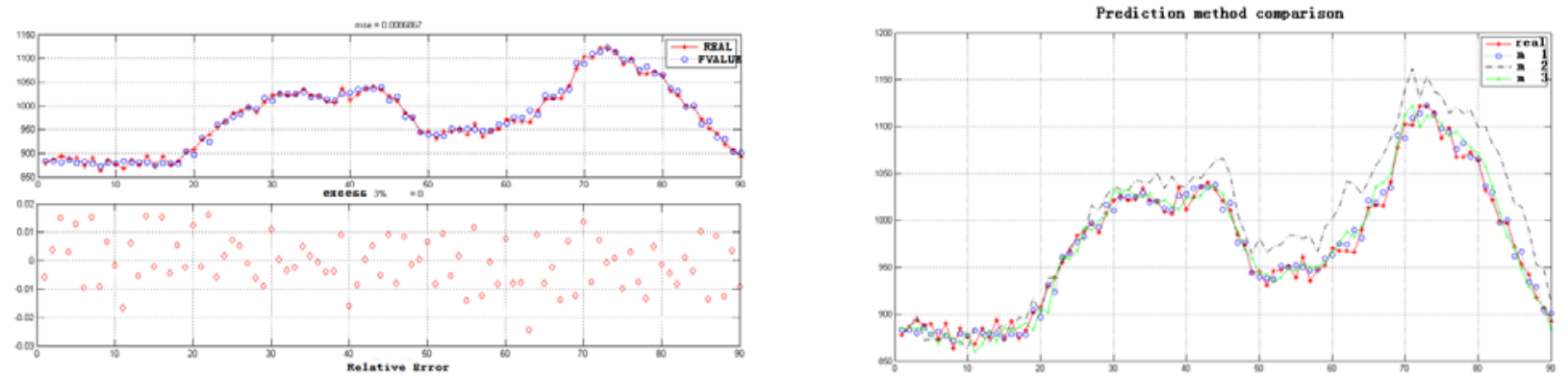

Fig.5. Forecast result of two types of signals Fig.6 Forecast result and relative error

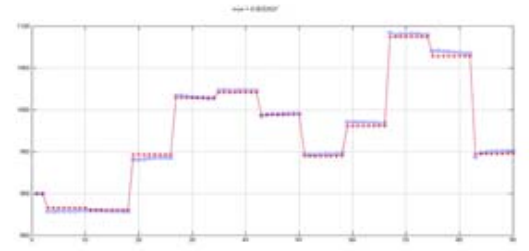

(a) approximation signal

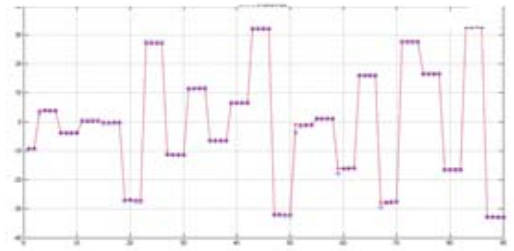

(b) three-layer detailed signal

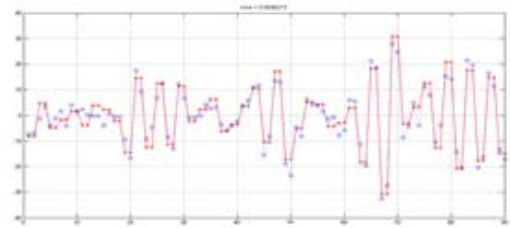

(c) fwo-layer detailed signal

Fig.7Comparison of three types of forecast

Figure 5 shows the forecast result of three-layer decomposition signal of this place on March 1, 2012 with the method described in this paper.

Here in consideration that the signal frequency in scale 1 is relatively high and the amplitude is not more than $3 \%$ of the total load basically, the first layer of detailed signal will be processed as interference signal. The input parameters are the data of first 6 points of the forecast points and the 
output is the forecast result of forecast point obtained through the model training.

Tab.1.Data comparison of forecast result

\begin{tabular}{lllll}
\hline Method & MAX & MIN & MEAN & MSE \\
\hline Method 1 & 0.0243 & 0.0037 & 0.0072 & 0.0087 \\
Method 2 & 0.0776 & 0.0026 & 0.0270 & 0.0327 \\
Method 3 & 0.0299 & 0.0009 & 0.0098 & 0.0120 \\
\hline
\end{tabular}

Figure 6 shows the comparison after linear superposition of daily load forecast result of the above two types of signal with the actual load as well as the relative error in each point.

Figure 7 is the comparison diagram of three different forecast methods. Method 1: adopt the multi-scale SVM forecast method described in this paper; method 2: multi-scale SVM forecast method without adding wavelet analysis; method 3: single-scale SVM forecast method with wavelet added.

Table 1 shows some data of the three forecast methods. It can be seen from Table 1 that compared with method 2, the load forecast based on wavelet analysis, the load forecast result is basically consistent with the actual load trend and the error is relatively small, indicating that wavelet analysis can effectively relieve the influence brought by the electric railway. Compared with method 3, the multi-scale SVM model is better than the single-scale SVM model in maximum relative error (MAX), mean relative error (MEAN) and(MSE) parameter, although the minimum relative error (MIN) is inferior to the single-scale SVM model, in consideration that there is a relatively strong contingency in this parameter and the $0.37 \%$ error is far less than $3 \%$ required in actual production, so this method can effectively improve the overall forecast precision under the influence of electric railway.

\section{Conclusion}

Against the problem of poor load forecast precision under the influence of electric railway, in this paper, we introduce wavelet analysis theory into the data processing for the first time and use wavelet function to divide the original data into appropriation and detailed parts, effectively controlling the prevision of load forecast result. Then through the method of improving the kernel function of support vector machine, we further improve the progress of ultrashort-term load forecast. A very good forecast result is obtained through combination of the two methods. After comparison with many forecast methods, the experiment result shows that this method is simple, reliable and practical and the forecast result conforms to the actual operation law, with precision improved by $2 \%$ at least. For the special load similar to the electric railway load, for example, large iron and steel enterprises and coal enterprises, the method described in this paper also has a certain reference value.

\section{References}

[1] Tomonobu S, Hitoshi T, Katsumi U, et al. One-Hour-Ahead Load Forecasting Using Neural Networks [J]. IEEE TRANSACTIONSONPOWER SYSTEMS, 2002,17 (1):113-118.

[2] Vermaak J ,Botha E C. Recurrent neutral network for short2term load forecasting [ J ] . IEEE Trans on Power System , 1998 , 13(1) :126-132.

[3] Lin Jipeng, Liu Junha, Research on Wavelet-based Support Vector Machine [J], Journal of Xi'an Jiaotong University, 2005, 39(8): 816-819.

[4] Zhang Li, Zhou Weida, Jiao Licheng. Wavelet Support Vector Machine [J]. IEEE Trans. on Systems, Man, and Cybernetics , Part B: Cybernetics, 2004, 34(1): 34-39.

[5] Dong sheng, YI Xuming.Wavelet de-noising based on four improved functions for threshold estimation. Journal of Mathematics. 2006.

[6] Zhang Ying, Forecast Method of Similar Day-based Neural Network Short-term Load 
[J]Heilongjiang University (Journal of Natural Science), 2003,20 (2): 59-61.nce.1065467.

[7] Tang Yin, Research on Time Sequence Similarity Analysis Method [J], Computer Engineering and Application, 2006, (1): 68-71

[8] Leontaritis I J, Billings S A. Input-output parametric models for nonlinear system s, part 1: deterministic nonlinear system s[ J] . Int J Con tr, 2006, 41 ( 2) : 303-3441.

[9] Nishiwaki K,Kagami S. High Frequency Walking Pattern Generation based on Preview Control of ZMP, IEEE International Conference on Robotics and Automation. 2006.

[10] Chen Nan and so on. For the field of industrial and highly interconnected, TI launched a new Sitara ARM9 microprocessor. [J]. Global Electronics, 2010 (5) 86-87.

[11] YUAN Xiao-fang, WANG Yao-nan. Selection of SVM parameters using chaos optimization algorithms[J].Control and Decision, 2006, 21 (1) : 111-115. 